\title{
Brain nitric oxide production by a proline-rich decapeptide from Bothrops jararaca venom improves baroreflex sensitivity of spontaneously hypertensive rats
}

\author{
Claudiana Lameu ${ }^{1,2}$, Vera Pontieri ${ }^{2}$, Juliano R Guerreiro ${ }^{3}$, Eduardo F Oliveira ${ }^{2,4}$, Carlos Alberto da Silva ${ }^{5}$, \\ Joyce M Giglio ${ }^{2}$, Robson L Melo ${ }^{2}$, Ruy R Campos ${ }^{6}$, Antonio Carlos Martins de Camargo ${ }^{1,7}$ and \\ Henning Ulrich ${ }^{1}$
}

Baroreflex sensitivity is disturbed in many people with cardiovascular diseases such as hypertension. Brain deficiency of nitric oxide (NO), which is synthesized by NO synthase (NOS) in the citrulline-NO cycle (with argininosuccinate synthase (ASS) activity being the rate-limiting step), contributes to impaired baroreflex. We recently showed that a decapeptide isolated from Bothrops jararaca snake venom, denoted Bj-PRO-10c, exerts powerful and sustained antihypertensive activity. Bj-PRO-10c promoted vasodilatation dependent on the positive modulation of ASS activity and NO production in the endothelium, and also acted on the central nervous system, inducing the release of GABA and glutamate, two important neurotransmitters in the regulation of autonomic systems. We evaluated baroreflex function using the regression line obtained by the best-fit points of measured heart rate (HR) and mean arterial pressure (MAP) data from spontaneously hypertensive rats (SHRs) treated with Bj-PRO-10c. We also investigated molecular mechanisms involved in this effect, both in vitro and in vivo. Bj-PRO-10c mediated an increase in baroreflex sensitivity and a decrease in MAP and HR. The effects exerted by the peptide include an increase in the gene expression of endothelial NOS and ASS. Bj-PRO-10c-induced NO production depended on intracellular calcium fluxes and the activation of a $\mathrm{G}_{\mathrm{i} / \mathrm{o}}$-protein-coupled metabotropic receptor. Bj-PRO-10c induced NO production and the gene expression of ASS and endothelial NOS in the brains of SHRs, thereby improving baroreflex sensitivity. Bj-PRO-10c may reveal novel approaches for treating diseases with impaired baroreflex function.

Hypertension Research (2010) 33, 1283-1288; doi:10.1038/hr.2010.208

Keywords: argininosuccinate synthase; baroreflex sensitivity; Bothrops jararaca venom; nitric oxide; proline-rich oligopeptide

\section{INTRODUCTION}

The gaseous messenger NO is involved mainly in the regulation of local and systemic vascular resistance, sodium balance and, consequently, blood pressure control, ${ }^{1}$ but it is also a signaling molecule and modulator of brain function. ${ }^{2}$ Recent studies relate NO with central cardiovascular control through the regulation of the cardiac and vascular autonomic system via the modulation of the central sites of cardiovascular autonomic neural integration. ${ }^{3,4}$

$\mathrm{NO}$ is generated in the citrulline-NO cycle by NO synthase (NOS) using L-arginine as a substrate. Three isoforms of NOS have been described: calcium-dependent endothelial (eNOS) and neuronal (nNOS) isoforms and inducible NOS. The expression and activity of the latter are induced by inflammatory stimuli, independent of the cytosolic calcium concentration. ${ }^{5}$

In the citrulline-NO cycle, argininosuccinate synthase (ASS) activity is rate-limiting with regard to the supply of L-arginine as substrate for NOS. ASS is an ubiquitous enzyme that is expressed in all tissues, including the brain. ${ }^{6}$ This enzyme catalyzes the conjugation reaction of citrulline with aspartate to generate argininosuccinate, which in turn is converted by argininosuccinate lyase into fumarate and $\mathrm{L}$-arginine. L-arginine is cleaved into citrulline and $\mathrm{NO}$, completing the cycle. ${ }^{6,7}$

Our group has recently shown that a decapeptide that is rich in proline residues binds to ASS. This molecule, named Bj-PRO-10c

${ }^{1}$ Departamento de Bioquímica, Instituto de Química, Universidade de São Paulo, São Paulo, Brazil; ${ }^{2}$ Center for Applied Toxinology-CAT/CEPID, Instituto Butantan, São Paulo, Brazil; ' ${ }^{2}$ aboratório de Bioquímica de Peptídeos e Proteínas, Departamento de Ciências Biológicas, ESALQ/USP, São Paulo, Brazil; ${ }^{4}$ Departamento de Bioquímica, Universidade Federal de São Paulo, São Paulo, Brazil; ${ }^{5}$ Natural and Human Sciences Center, Universidade Federal do FABC, Santo André, Brazil; ${ }^{6}$ Department of Physiology, Universidade Federal de São Paulo, São Paulo, Brazil and 7Department of Cell Biology and Development, Universidade de São Paulo, São Paulo, Brazil

Correspondence: Dr H Ulrich, Departamento de Bioquímica, Instituto de Química, Universidade de São Paulo, Avenida Professor Lineu Prestes 748, São Paulo 05508-900, Brazil. E-mail: henning@iq.usp.br

Received 9 June 2010; revised 28 July 2010; accepted 7 August 2010 
or BPP-10c, belongs to a class of peptides isolated from Bothrops jararaca snake venom. These peptides are products of C-type natriuretic peptide precursor protein cleavage in the venom gland and the brain of the snake $\mathrm{e}^{8,9} \mathrm{Bj}$-PRO-10c exerted powerful and sustained antihypertensive activity ${ }^{10}$ and vasodilatation depended on the positive modulation of ASS activity and NO production in the endothelium. ${ }^{11}$

Changes in mean arterial pressure (MAP) promoted by $\mathrm{Bj}-\mathrm{PRO}-10 \mathrm{c}$ are accompanied by a significant reduction in heart rate (HR),${ }^{10}$ rather than by an increase in HR as would be expected by the discharge of baroreceptors due to hypotension. ${ }^{12}$ These results, together with the observation that about $1 \%$ of $B j$-PRO- $10 \mathrm{c}$ administered i.v. to mice is found in the brains of these animals, ${ }^{13}$ suggest that this peptide affects the central nervous system (CNS). Recently, we have shown that $B j-P R O-10 c$ promotes the release of the neurotransmitters GABA and glutamate, which are known to participate in the regulation of cardiac and vascular autonomic systems, thus leading to a decline of MAP and HR among spontaneously hypertensive rats (SHRs). ${ }^{14}$ Therefore, $B j$-PRO-10c-induced decreases of MAP and HR could result from interference of $B j-\mathrm{PRO}-10 \mathrm{c}$ with the autonomic nervous system, provoking changes in HR and baroreflex control. ${ }^{15,16}$

Arterial baroreflex is one of the most important regulatory mechanisms in the cardiovascular system. Baroreflex function, expressed as baroreflex sensitivity, is measured to assess autonomic control. Clinical evidence has associated arterial baroreflex dysfunction with many cardiovascular diseases, the sudden death of patients suffering acute myocardial infarction and mortality in patients with congestive heart failure. ${ }^{17}$ Changes in neural-cardiovascular integration due to NO deficiency may be the main cause of autonomic abnormalities in several models of experimental hypertension ${ }^{2}$ as a consequence of impaired arterial baroreflex. ${ }^{3,18}$

As a result of augmented ASS activity, an enzyme also expressed in the CNS, ${ }^{6}$ and central effects on the control of blood pressure ${ }^{11,14}$ in the presence of $B j-\mathrm{PRO}-10 \mathrm{c}$, we expected to observe increased NO production in the brain and improved arterial baroreflex function in conscious SHRs. Therefore, this study was designed to examine the effects of Bj-PRO-10c on baroreflex sensitivity in SHRs and to elucidate involved mechanisms.

\section{METHODS}

\section{Synthesis and purification of $\mathrm{Bj}$-PRO-10c}

Synthesis of $B j$-PRO-10c ( $<$ ENWPHPQIPP) was performed as detailed elsewhere. ${ }^{14,19}$ Briefly, the synthesis of Bj-PRO-10c was carried out on an automated PSSM-8 peptide synthesizer (Shimadzu, Chiyoda-ku, Kyoto, Japan) by a stepwise solid-phase method using $N$-9-fluorenylmethoxycarbonyl chemistry (Novabiochem-EMD Chemicals, San Diego, CA, USA). The crude synthetic peptide was purified by preparative reverse-phase high-performance liquid chromatography. Buffer elution residues were removed by freeze drying, followed by confirmation of sequence identity and purity by analytical highperformance liquid chromatography and MALDI-TOF mass spectrometry (Amersham Biosciences, Uppsala, Sweden).

\section{Collection of brains of $B$ j-PRO-10c-treated rats}

Male SHRs (270-350 g) from the animal house of the Instituto de Ciências Biológicas (University of São Paulo, Brazil) and normotensive Wistar rats (270-350 g) from the Butantan Institute were used to obtain the brains for determination of NO production and mRNA transcription levels. The animals were kept in a 12:12 light/dark cycle with standard laboratory chow and water provided ad libitum. All surgical procedures and protocols were in accordance with Guidelines for Ethical Care of Experimental Animals and had been previously approved by the Institutional Animal Care and Use Committee of the Butantan Institute. Cardiovascular parameters of Wistar rats and SHRs were monitored for $6 \mathrm{~h}$ following i.v. injection of $71 \mathrm{nmol} \mathrm{kg}{ }^{-1} B j$-PRO-10c or saline as detailed by Ianzer $e$ et al. ${ }^{10}$ Then, animals were anesthetized by injection of $10 \%$ ketamine and $2 \%$ xylazine (1:1) in a volume of $50 \mu$ followed by intracardiac perfusion (from the left ventricle and through the right atrium) with $20 \mathrm{ml}$ of $0.15 \mathrm{M} \mathrm{NaCl}$ and $0.01 \%$ sodium heparin at a flow rate of $4 \mathrm{ml} \mathrm{min}{ }^{-1}$. Brain tissue was immediately removed and frozen to $-80^{\circ} \mathrm{C}$ until further use.

\section{Preparation of rat brain cell culture}

Whole-brain primary neuronal culture was prepared according to Lameu et al. ${ }^{14}$ Cells obtained from dissected brain following removal of pia mater and blood vessels were dissociated in the presence of trypsin and the cell suspension was plated onto poly-L-lysine-precoated tissue culture dishes in Dulbecco's modified Eagle's medium containing 10\% horse serum (Gibco BRL, Grand Island, NY, USA), as previously described. ${ }^{20}$ Following 3 days of culture, $10 \mu \mathrm{M}$ cytosine-arabinoside was added to eliminate cells of non-neuronal origin. ${ }^{21}$ Cells were cultured for $10-15$ days at $37^{\circ} \mathrm{C}$ in a water-saturated atmosphere containing $5 \% \mathrm{CO}_{2}$.

\section{Chemiluminescence assay for the measurement of NO products}

$\mathrm{NO}$ is a gaseous molecule with an extremely short half-life. It is rapidly converted into NO-derived products (NOx), such as nitrate, nitrite, nitrosothiol, nitrosamines and iron-nitrosyl complexes. ${ }^{22}$ For the determination of NOx concentrations in whole-brain homogenates, extra and intracellular media of rat brain cell culture were analyzed with a chemiluminescence Sievers Nitric Oxide Analyzer-NOA 280i (GE Analytical Instruments, Boulder, CO, USA), following the procedure optimized by Feelisch et al..$^{22}$ Whole brains of SHRs treated with $71 \mathrm{nmol} \mathrm{kg}^{-1} \mathrm{Bj}$-PRO-10c or vehicle control (saline) were homogenized in RIPA buffer ( $50 \mathrm{~mm}$ Tris pH 7.5, $150 \mathrm{~mm} \mathrm{NaCl}, 1 \%$ Nonidet p-40, $0.5 \%$ sodium deoxycholate, $0.1 \%$ sodium dodecyl sulfate, $1 \mathrm{~mm}$ diethylenetriamine pentaacetic acid and $10 \mathrm{~mm} N$-ethylmaleimide). In all experiments, $1 \mathrm{ml}$ of extracellular cell culture medium was treated with a concentrated solution of $\mathrm{N}$-ethylmaleimide to give a final concentration of $10 \mathrm{~mm}$. Cells were washed with phosphate-buffered saline and lysed in RIPA buffer. Cell lysates were collected and incubated for $30 \mathrm{~min}$ on ice and centrifuged for $15 \mathrm{~min}$ at $21000 \mathrm{~g}$ and $4{ }^{\circ} \mathrm{C}$. The collected supernatant contained the intracellular medium. Immediately after the cell lysis procedure, samples were directly injected into a vessel containing a saturated solution of vanadium (III) chloride in $1 \mathrm{~N} \mathrm{HCl}$ at $90^{\circ} \mathrm{C}$ for NOx concentration measurements. Under these conditions, all NOx metabolites were reduced and compared with those of standard nitrate solutions. $^{22}$

\section{Real-time PCR}

Total RNA was extracted from the brains of SHRs using Trizol (Life Technologies, Gaithersburg, MD, USA). Every sample was further treated with Amplification Grade DNase I (Sigma Chemical, Poole, UK). An aliquot of the sample was used to confirm the absence of any residual DNA that could generate false-positive results. Reverse transcription reactions for cDNA synthesis were performed with a thermal cycler (Applied Biosystems, Foster City, CA, USA) using the SuperScript III First-Strand synthesis system according to the manufacturer's protocol (Invitrogen, Carlsbad, CA, USA), in the presence of primers specific for ASS- , eNOS- and nNOS-coding sequences. The expression levels of selected mRNAs were measured by real-time PCR using the ABI Step One Plus Instrument (Applied Biosystems). PCR reactions were performed in $25 \mu \mathrm{l}$ of buffer containing $1 \mu \mathrm{l}$ of cDNA, SYBR Green Master Mix (Applied Biosystems) and $5 \mathrm{pmol}$ of sequence-specific primer: ASS (forward primer 5'-TGCACTCTATGAGGACCGCTATC-3', reverse primer 5'-CTAGGCACCTC TCTCGCCAGGCCT-3'); eNOS (forward primer $5^{\prime}$-GACTTTTAAGGAAGT AGCCAATGCA-3', reverse primer $5^{\prime}$-CCATACAGGATAAGTCGCCTTCAC-3'); nNOS (forward primer 5'-CCAATGTTCACAAAAAACGAGTCT-3', reverse primer 5'-TCGCCTGGACTTAGGGCTTT-3') and glyceraldehyde 3-phosphate dehydrogenase (forward primer 5'-TGGCCTCCAAGGAGTAAGAAAC-3', reverse primer $5^{\prime}$-GGCCTCTCTCTTCCTCTCAGTATC- $3^{\prime}$ ). Thermal cycling conditions consisted of a preincubation step for $2 \mathrm{~min}$ at $50^{\circ} \mathrm{C}$, then denaturation for $10 \mathrm{~min}$ at $95^{\circ} \mathrm{C}$ followed by 40 cycles of denaturation for $15 \mathrm{~s}$ at $95^{\circ} \mathrm{C}$ and annealing/extension for $1 \mathrm{~min}$ at $60^{\circ} \mathrm{C}$. The comparative $2^{-\Delta \Delta C \mathrm{~T}}$ method 
was used for the relative quantification of gene expression as described previously. ${ }^{23}$

\section{Baroreceptor reflex assay}

Normotensive Wistar rats and SHRs (270-350 g) were anesthetized with $0.05 \mathrm{ml}$ of $10 \%$ ketamine and $2 \%$ xylazine (1:1), and catheters (TYGON Flexible Plastic Tubing, Saint-Gobain Performance Plastics Corporation, New Haven, CT, USA) were inserted into the femoral artery and vein for cardiovascular monitoring and drug administration. The catheters were tunneled subcutaneously to the back of neck, where they were fixed. Following recovery from anesthesia for 1 day, both MAP and HR were recorded continuously in freely moving rats using BIOPAC Systems instrumentation (BIOPAC Systems, Goleta, CA, USA). After recording the basal values of MAP and HR, control measurements of MAP and HR were followed by repeated stimulation of baroreceptors with $0.1 \mathrm{ml}$ bolus injections of graded doses of phenylephrine $\left(0.1-6.4 \mu \mathrm{g} \mathrm{kg}^{-1}\right)$ and sodium nitroprusside $\left(0.2-12.8 \mu \mathrm{g} \mathrm{kg}^{-1}\right)$ into the femoral vein. Phenylephrine and sodium nitroprusside injections were given in a random order, and subsequent stimulations were not applied until the recorded parameters had returned to preinjection levels. The peak increase or decrease in MAP after each injected dose of phenylephrine and sodium nitroprusside was correlated with the peak reflex change in HR. Average values obtained during a fixed time interval were used to quantify reflex changes. Baroreflex sensitivity was analyzed by the regression line obtained by best-fit points to measured HR (bpm) and MAP (mm Hg) data. ${ }^{24}$

\section{Statistical analysis}

Statistical analysis was conducted using one-way ANOVA, employing the GraphPad3 software. Differences were considered significant when $P<0.05$.

\section{RESULTS}

\section{$B j$-PRO-10c-induced NO production in the brains of SHRs}

The Bj-PRO-10c action on NO production in rat brains was determined by measuring $\mathrm{NO}$ metabolites $(\mathrm{NOx}=$ nitrate, nitrite, nitrosothiol, nitrosamines and iron-nitrosyl complexes) in primary brain cell cultures of SHRs and whole brains of normo- and hypertensive rats in the presence and absence of the peptide. The concentration of NOx in the brains of SHRs was $93.5 \pm 18.5 \mu \mathrm{m}$ following $6 \mathrm{~h}$ of treatment with $71 \mathrm{nmol} \mathrm{kg}^{-1} B j-\mathrm{PRO}-10 \mathrm{c}$, which was $34 \%$ higher than the NOx content of control rats $(69.3 \pm 12.1 \mu \mathrm{M})$. In the brains of normotensive rats, the NOx content did not change following treatment with the same concentration of Bj-PRO-10c (Figure 1). $B j$-PRO-10c also led to an increase in NOx concentration in cell culture from $0.38 \pm 0.01 \mu \mathrm{M}$ to $1.04 \pm 0.04 \mu \mathrm{M}$ (Figure 2). For the elucidation of $B j-\mathrm{PRO}-10 \mathrm{c}$-mediated signal transduction, cells were exposed for $24 \mathrm{~h}$ to $1 \mu \mathrm{M} B j$-PRO-10c in the absence or presence of $100 \mathrm{ng} \mathrm{ml}^{-1}$ pertussis toxin (an inhibitor of $\mathrm{G}_{\mathrm{i} / \mathrm{o}}$-protein-coupled metabotropic receptor activation), $10 \mathrm{~mm}$ ethylene glycol tetraacetic acid (EGTA; to chelate extracellular calcium), or $200 \mathrm{ng} \mathrm{ml}^{-1}$ thapsigargin (an inhibitor of endoplasmic reticulum $\mathrm{Ca}^{2+}$-ATPase). ${ }^{25}$ $B j$-PRO-10c-evoked NOx production was reduced by thapsigargin and EGTA to $0.39 \pm 0.01$ and $0.59 \pm 0.09 \mu \mathrm{M}$, respectively. As a result of these studies, both calcium influx and the mobilization of intracellular calcium stores contributed to Bj-PRO-10c-induced signaling and NO production. Pertussis toxin also interfered with NO production, suggesting the participation of a $\mathrm{G}_{\mathrm{i} / \mathrm{o}}$-protein-coupled metabotropic receptor in the $B j-\mathrm{PRO}-10 \mathrm{c}$-induced signaling pathway (Figure 2).

\section{Regulation of ASS and eNOS gene expression in SHR brain by $B j$-PRO-10c}

We have analyzed the gene expression of the enzymes ASS, eNOS and nNOS, which are known to be involved in NO synthesis. Real-time PCR revealed that Bj-PRO-10c mediated $2.2 \pm 0.8$ - and $1.9 \pm 0.34$-fold

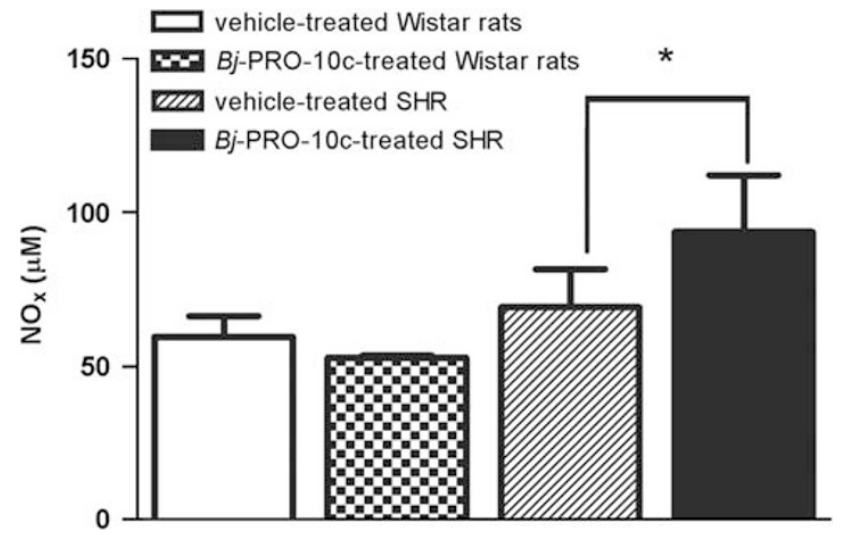

Figure 1 NO concentration in the brain of normotensive (Wistar) and hypertensive rats (SHRs) treated with Bj-PRO-10c. Conscious rats $(n=4)$ were treated with $71 \mathrm{nmol} \mathrm{kg}^{-1}$ Bj-PRO-10c or saline i.v. After $6 \mathrm{~h}$ of treatment, animals were anesthetized and then the brain was removed and homogenized in RIPA buffer. The homogenate was used to measure the NO concentration by using a chemiluminescence assay. The data are presented as mean values \pm s.e. compared with the vehicle-treated rats, for a period up to $6 \mathrm{~h}$. ${ }^{*} P<0.05$.

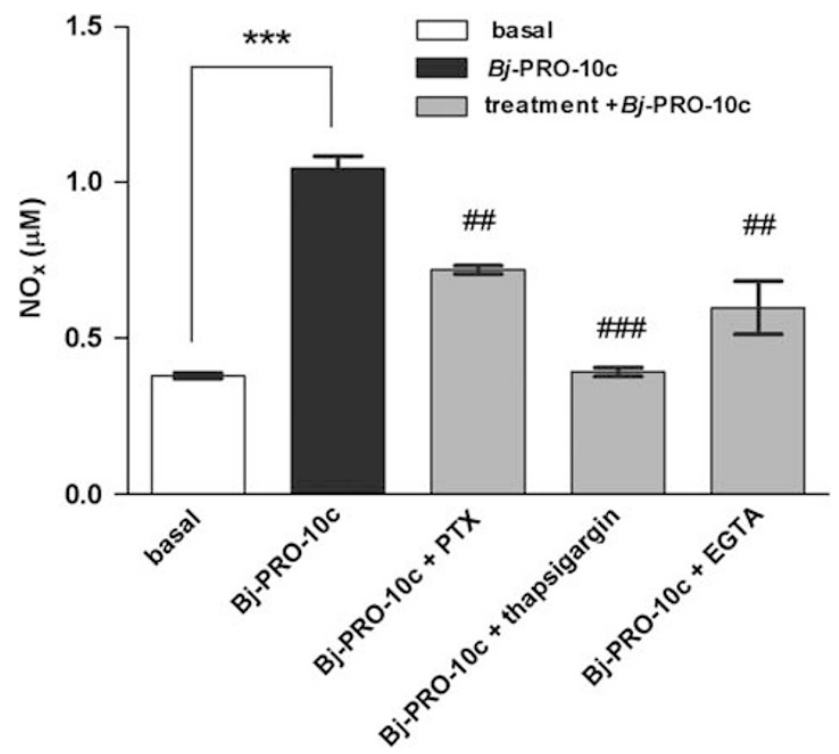

Figure 2 Bj-PRO-10c induced NO metabolites production in neuronal cells. Neuronal cells obtained from postnatal (1-day-old) SHRs were seeded into poly-D-lysine-coated Petri dishes in appropriate medium and incubated for $24 \mathrm{~h}$. Afterwards, cells were kept for $24 \mathrm{~h}$ in serum-free medium containing $1 \mu \mathrm{m}$ of Bj-PRO-10c. The medium was collected and the cells were lysed with RIPA buffer for measuring NO products in extracellular and intracellular media by a chemiluminescence assay. Bj-PRO-10c-induced NO production was also determined in the presence of $100 \mathrm{ng} \mathrm{ml}^{-1}$ pertussis toxin (PTX), $10 \mathrm{~mm}$ EGTA or $200 \mathrm{ng} \mathrm{m}^{-1}$ thapsigargin. NO concentration was expressed as mean values \pm s.e. of six experiments. ${ }^{* *} P<0.001$ compared with basal NO levels; $\# \#<0.01$; \#\#\# $P<0.001$ compared with NO production in the presence of $B j$-PRO-10c.

increases in ASS and eNOS mRNA transcription levels, respectively, when compared with the gene expression rates of vehicle-treated control animals. However, nNOS expression in the brains of $B j$-PRO-10c-treated SHRs was not significantly different from that observed in the control group (Figure 3). 


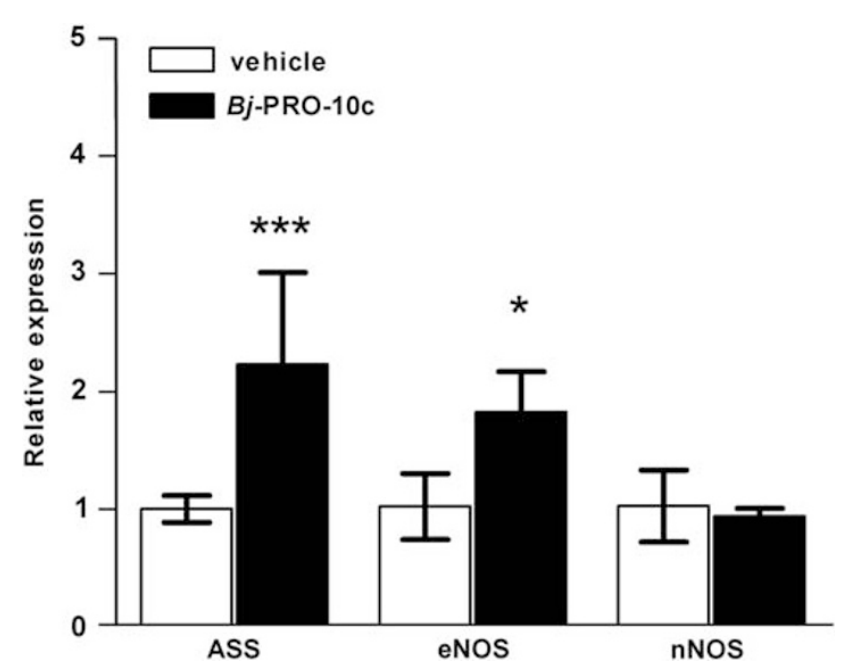

Figure 3 Regulation of ASS, eNOS and nNOS expression in SHR brain. SHRs were anesthetized $6 \mathrm{~h}$ after endovenous injection of $71 \mathrm{nmol} \mathrm{kg}^{-1}$ $B j$-PRO-10c and brains were removed for RNA extraction. Gene expression changes in SHR treated with Bj-PRO-10c brain was analyzed by quantitative real-time PCR and normalized using glyceraldehyde 3-phosphate dehydrogenase (GAPDH) gene expression as internal control. The obtained data were compared with gene expression levels of vehicle-treated SHRs. ${ }^{*} P<0.05$; $* * * P<0.001(n=4)$.

\section{Bj-PRO-10c improves baroreflex sensitivity of SHRs}

The baroreceptor reflex activities of conscious normotensive Wistar rats and SHRs were determined immediately, 1 or $4 \mathrm{~h}$ after the i.v. administration of $71 \mathrm{nmol} \mathrm{kg}^{-1} B j$-PRO-10c. The baroreflex sensitivity of SHRs analyzed by $\triangle \mathrm{HR} / \triangle \mathrm{MAP}$ indexes ( $b=$ best-fit slope) for bradycardia and tachycardia were $-1.7 \pm 0.3$ and $-0.5 \pm$ $0.1 \mathrm{bpm}(\mathrm{mm} \mathrm{Hg})^{-1}$, and those of normotensive rats were $-4.8 \pm 0.4$ and $-1.6 \pm 0.2 \mathrm{bpm}(\mathrm{mm} \mathrm{Hg})^{-1}$, respectively. These data are in agreement with a previous study by Krieger and co-workers demonstrating that SHRs have impaired sensitivity with regard to baroreflex control of $\mathrm{HR}^{26}$ After $\mathrm{Bj}$-PRO-10c injection, the baroreflex sensitivity of normotensive rats for the control of HR was unchanged (Figure 4a); however, baroreflex sensitivity increased among SHRs. The improvement of baroreflex sensitivity occurred only during periods of decreasing MAP (Table 1). One after Bj-PRO-10c injection, baroreflex sensitivity increased to $-0.7 \pm 0.1 \mathrm{bpm}(\mathrm{mm} \mathrm{Hg})^{-1}$. After $4 \mathrm{~h}$, the elevation of baroreflex sensitivity was even higher, reaching $-0.9 \pm 0.1 \mathrm{bpm}(\mathrm{mm} \mathrm{Hg})^{-1}$. No change was observed in tachycardic baroreflex following Bj-PRO-10c application (Figure $4 \mathrm{~b}$ ).

\section{DISCUSSION}

Our group, attempting to elucidate molecular mechanism of the antihypertensive activity of Bj-PRO-10c, identified ASS as a target for this peptide. ${ }^{11}$ ASS is the rate-limiting enzyme of the citrulline-NO cycle that regulates the supply of L-arginine as a substrate for NOS. ${ }^{6}$ We showed that Bj-PRO-10c administration results in augmented NO production in SHR brain tissues as well as in primary cultures of postnatal SHR brain neurons. Bj-PRO-10c-induced NO production in neuronal cells was abolished by pretreatment of cells with ASS and NOS inhibitors (data not shown). These results were in agreement with those obtained in endothelial cells. ${ }^{11}$ The results of the latter experiments suggested that $B j-\mathrm{PRO}-10 \mathrm{c}$-induced $\mathrm{NO}$ production involved calcium fluxes because both calcium signaling and NO production were affected by pretreatment with thapsigargin, EGTA
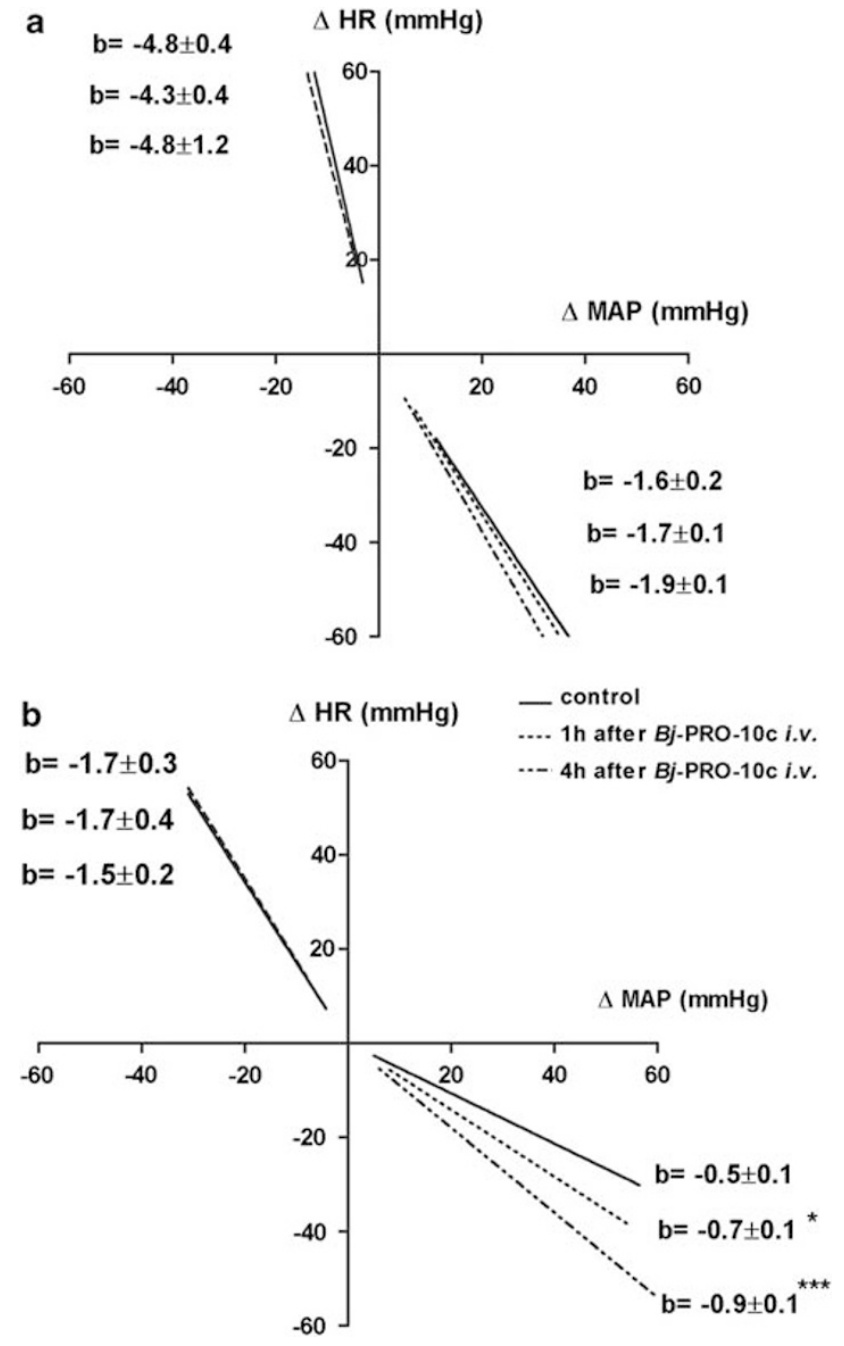

Figure 4 Baroreflex control of heart rate (HR) in rats. Average baroreflex linear curves obtained by means changes in heart rate $(<H R)$ due to induced changes in mean arterial pressure (MAP) $(<M A P)$ in normotensive (a) and spontaneously hypertensive (b) rats before (control), and 1 and $4 \mathrm{~h}$ after endovenous administration of $71 \mathrm{nmol} \mathrm{kg}^{-1} B j-P R O-10 \mathrm{c}$. Lines represent linear regression fitting of changes in HR and MAP. The slope of linear regressions $(b)$ was used to determine the gain of the baroreflex sensitivity. ${ }^{*} P<0.05$ and $* * * P<0.001$ compared with control (absence of $B j$-PRO-10c) ( $b=$ best-fit slope).

and pertussis toxin. These results are in agreement with previous research. ${ }^{14}$

NO has been attributed to various functions, including noncholinergic and non-adrenergic smooth muscle relaxation, the reduction of arterial pressure and signal transmission in the CNS. ${ }^{2}$ NO-mediated actions in the CNS include central vascular regulation ${ }^{27}$ and the baroreflex control of HR. ${ }^{18}$ Antihypertensive activity, based on the facilitated release of GABA and glutamate in the CNS, and NO production are suggested to result in diminished transmission of sympathetic tone to the periphery. ${ }^{27}$ However, no Bj-PRO-10cinduced changes of the sympathetic renal activity of SHRs were observed under anesthesia, and MAP of these animals also remained unchanged (data not shown). It is known that some anesthetics, such as pentobarbital, block the sympathetic nervous system ${ }^{28}$ and subsequently prevent changes in blood pressure, thereby explaining 
Table 1 Effects of $B j$-PRO-10c $\left(71 \mathrm{nmol} \mathrm{kg}^{-1}\right)$ on MAP, HR and baroreflex activity of normotensive rats and SHRs

\begin{tabular}{|c|c|c|c|c|}
\hline & $\begin{array}{l}\text { Baseline values } \\
\text { MAP ( } \mathrm{mm} \mathrm{Hg})\end{array}$ & $\begin{array}{c}\text { Baseline values } \\
\text { HR (bpm) }\end{array}$ & $\begin{array}{l}\text { Tachycardic gain } \\
\left(b p m\left(m m H^{-1}\right)^{-1}\right)\end{array}$ & $\begin{array}{l}\text { Bradycardic gain } \\
\left(b p m(m m H g)^{-1}\right)\end{array}$ \\
\hline \multicolumn{5}{|c|}{ Normotensive } \\
\hline Control & 120 & 347 & $-4.8 \pm 0.4$ & $-1.6 \pm 0.2$ \\
\hline $1 \mathrm{~h}$ & 117 & 326 & $-4.3 \pm 0.4$ & $-1.7 \pm 0.1$ \\
\hline $4 \mathrm{~h}$ & 118 & 363 & $-4.8 \pm 1.2$ & $-1.9 \pm 0.1$ \\
\hline \multicolumn{5}{|c|}{ Hypertensive } \\
\hline Control & 191 & 404 & $-1.7 \pm 0.3$ & $-0.5 \pm 0.1$ \\
\hline $1 \mathrm{~h}$ & 179 & 351 & $-1.7 \pm 0.4$ & $-0.7 \pm 0.1^{*}$ \\
\hline $4 \mathrm{~h}$ & 163 & 340 & $-1.5 \pm 0.2$ & $-0.9 \pm 0.1^{* * *}$ \\
\hline
\end{tabular}

Abbreviations: HR, heart rate; MAP, mean arterial pressure; SHR, spontaneously hypertensive rat. ${ }^{*} P<0.05$ and ${ }^{* *} P<0.001$ compared with control (absence of $B j-P R O-10 c$ ).

the inefficiency of $B j$-PRO-10c action under these experimental conditions. In addition, baroreflex sensitivity can be used as an index of autonomic control; thus, this observation provides further evidence for the participation of the sympathetic nervous system in $B j$-PRO-10c-mediated effects.

Recent evidence suggests that changes in arterial baroreflex control and the autonomous nervous system are involved in the development and maintenance of hypertension. ${ }^{29-31}$ In a classic study carried out in the 1960 's, Bristow et al. ${ }^{32}$ reported for the first time that the baroreflex control of HR in patients with hypertension was significantly lower than that of normotensive individuals. Herein, we have observed that baroreflex sensitivity in normotensive rats is much higher than in SHRs, which supports the previous study. ${ }^{26}$ Interventionist attitudes that seek improvement in arterial baroreflex sensitivity are considered to be new strategies for the management of cardiovascular diseases, especially hypertension. ${ }^{26,33}$

Recently, we published a study showing that Bj-PRO-10c mediated CNS actions to decrease arterial pressure and HR in SHRs. Bj-PRO10c-induced intracellular calcium signaling involved the activation of G-protein-coupled receptors and the release of neurotransmitters such as GABA and glutamate. ${ }^{14}$ Both GABA and glutamate participate in the regulation of vasodilatation through the reduction of sympathetic activity and baroreflex sensitivity control. ${ }^{34}$ Herein, we show that the i.v. application of Bj-PRO-10c resulted in augmented baroreflex sensitivity. We observed that baroreflex sensitivity was most increased after $4 \mathrm{~h}$ of treatment with Bj-PRO-10c. However, the effects exerted by this peptide were less pronounced at $1 \mathrm{~h}$ after exposure to $B j$-PRO10c. These observations may explain the moderate reduction in arterial pressure that occurs after the first hour and the higher and prolonged rise in arterial pressure that occurs $3 \mathrm{~h}$ after the i.v. injection of the peptide. ${ }^{10}$ Furthermore, no effect on baroreflex sensitivity was observed in normotensive rats, just as significant changes in MAP and HR of normotensive rats were not previously found after administration of $B j$-PRO- $10 c^{10}$ (Table 1).

Baroreflex function, which has an important role in regulating blood pressure, in both the short and long term, is influenced by genetic factors. ${ }^{35}$ Xing-Sheng et al. ${ }^{31}$ concluded from human genetic studies that polymorphism of the eNOS gene results in reduced eNOS activity, indicating a depressant effect of eNOS-generated NO on baroreflex sensitivity. Based on the observation that Bj-PRO-10c induced production of $\mathrm{NO}$ in neuronal cells from postnatal whole-
SHR-brain cell culture, we analyzed the gene expression levels of ASS, eNOS and nNOS, which are known to be involved in NO synthesis. $B j$-PRO-10c treatment induced an increase in ASS gene expression; ASS is an essential enzyme in the citrulline-NO cycle that promotes the production of L-arginine as a natural, unique substrate for NOS. A previous study showed that Bj-PRO-10c potentiated ASS activity in vitro and in vivo, resulting in increased intracellular L-arginine concentration. ${ }^{11}$ Endogenous L-arginine synthesis, as part of the citrulline-NO cycle, is crucial for NO production because most of the ingested L-arginine is degraded by liver arginase. ${ }^{36}$ ASS is widely distributed throughout the brain and interneurons, and it has possible neuromodulatory effects; ${ }^{37}$ however, this is the first time that evidence has been provided for the involvement of ASS in the central control of blood pressure.

In contrast, nNOS was not differentially expressed in Bj-PRO-10ctreated SHR brains when compared with vehicle-treated animals. However, the gene expression levels of eNOS, similar to those of ASS, were also augmented in the brains of animals treated with the peptide. Our data agree with the results obtained by Kishi et al. ${ }^{38}$ showing that the overexpression of eNOS in the rostral ventrolateral medulla and the nucleus of the solitary tract of hypertensive and Wistar rats results in reduced systolic arterial pressure and reduced HR. However, the decrease in arterial pressure was more pronounced in SHRs when compared with Wistar rats with normal blood pressure. ${ }^{38}$ It has been suggested that the development of hypertension in SHRs involves abnormalities in the citrulline-NO cycle. ${ }^{39}$ Although high concentrations of nNOS are present in the CNS and principally in nucleus of the solitary tract and rostral ventrolateral medulla, its expression levels do not differ between SHRs and rats with normal arterial pressures. ${ }^{39}$ This observation suggests that there are other compensatory mechanisms for the reduction of arterial pressure that do not involve nNOS activity. The reduction of blood pressure following eNOS activation may be due to decreased sympathetic nerve activity. ${ }^{39}$

In summary, the increased expression of eNOS led to augmented baroreflex sensitivity in the brains of hypertensive rats and reduced MAP and $\mathrm{HR}^{40}{ }^{40}$ as observed in our studies following Bj-PRO-10c administration. These effects result from increased eNOS expression and appear to depend on NO-induced liberation of GABA. ${ }^{38}$ In support of such a mechanism, Bj-PRO-10c also provoked GABA release in brain cell culture ${ }^{14}$ in addition to diminishing MAP and $\mathrm{HR}$ and increasing baroreflex sensitivity, as shown in the present study.

We have described a new mechanism of action for Bj-PRO-10c previously known for its strong angiotensin I converting enzyme inhibitor properties. Through this mechanism, Bj-PRO-10c induces NO production which has key roles in the baroreflex control mechanism. Most importantly, the dose of $B j-\mathrm{PRO}-10 \mathrm{c}$ required to effectively increase baroreflex sensitivity and induce changes in NO production and mRNA transcription levels is not sufficient for angiotensin I converting enzyme inhibition in vivo. ${ }^{10}$

The fact that Bj-PRO-10c interacts with ASS, a target that is untapped by the pharmaceutical industry, and modulates gene expression of key enzymes in NO production opens avenues for its use as a structural model for the development of novel pharmacological agents for treating diseases characterized by impaired baroreflex function.

\section{CONFLICT OF INTEREST}

The authors declare no conflict of interest. 


\section{ACKNOWLEDGEMENTS}

H.U. and A.C.M.C. are grateful for grant support from Fundação de Amparo à Pesquisa do Estado de São Paulo (FAPESP) and Conselho Nacional de Desenvolvimento Científico e Tecnológico (CNPq), Brazil. J.R.'s and C.L.'s doctoral theses were supported by a fellowship from FAPESP. J.R. and C.L. are currently supported by postdoctoral fellowships from FAPESP. J.G.'s and E.F.O.'s master's thesis research was supported by fellowships from FAPESP. K.L.P.M's master's thesis was supported by CNPq. Maria Aparecida Siqueira is acknowledged for technical support.

1 Umans JG, Levi R. Nitric oxide in the regulation of blood flow and arterial pressure. Annu Rev Physiol 1995; 57: 771-790.

2 Garthwaite J, Boulton CL. Nitric oxide signaling in the central nervous system. Annu Rev Physiol 1995; 57: 683-706.

3 Souza HC, De Araújo JE, Martins-Pinge MC, Cozza IC, Martins-Dias DP. Nitric oxide synthesis blockade reduced the baroreflex sensitivity in trained rats. Auton Neurosci 2009; 150: 38-44.

4 Martins-Pinge MC, Garcia MR, Zoccal DB, Crestani CC, Pinge-Filho P. Differential influence of iNOS and nNOS inhibitors on rostral ventrolateralmedullary mediated cardiovascular control in conscious rats. Auton Neurosci 2007; 131: 65-69.

5 Galea E, Feinstein DL, Reis DJ. Induction of calcium-independent nitric oxide synthase activity in primary rat glial cultures. Proc Natl Acad Sci USA 1992; 89: 10945-10949.

6 Husson A, Brasse-Lagnel C, Fairand A, Renouf S, Lavoinne A. Argininosuccinate synthetase from the urea cycle to the citrulline-NO cycle. Eur J Biochem 2003; 270: 1987-1999

7 Flam BR, Hartmann PJ, Harrell-Booth M, Solomonson LP, Eichler DC. Caveolar localization of arginine regeneration enzymes, argininosuccinate sythase, and lyase, with endothelial nitric oxide synthase. Nitric Oxide 2001; 5: 187-197.

8 Murayama N, Hayashi MA, Ohi H, Ferreira LA, Hermann VV, Saito H, Fujita Y, Higuchi $\mathrm{S}$, Fernandes BL, Yamane T, de Camargo AC. Cloning and sequence analysis of a Bothrops jararaca cDNA encoding a precursor of seven bradykinin-potentiating peptides and a C-type natriuretic peptide. Proc Natl Acad Sci USA 1997; 94: 1189-1193.

9 Hayashi MA, Murbach AF, lanzer D, Portaro FC, Prezoto BC, Fernandes BL, Silveira PF, Silva CA, Pires RS, Britto LR, Dive V, Camargo AC. The C-type natriuretic peptide precursor of snake brain contains highly specific inhibitors of the angiotensin-converting enzyme. J Neurochem 2003; 85: 969-977.

10 lanzer D, Santos RA, Etelvino GM, Xavier CH, de Almeida Santos J, Mendes EP, Machado LT, Prezoto BC, Dive V, de Camargo AC. Do the cardiovascular effects of angiotensin-converting enzyme (ACE) I involve ACE-independent mechanisms? new insights from proline-rich peptides of Bothrops jararaca. J Pharmacol Exp Ther 2007; 322: 795-805.

11 Guerreiro JR, Lameu C, Oliveira EF, Linares E, Augusto O, Lebrun I, Serrano SMT, Camargo ACM. Argininosuccninate synthase (AsS) is a novel target for a snake venom anti-hypertensive oligopeptide. J Biol Chem 2009; 284: 20022-20033.

12 Bunag RD, Walaszek EJ, Mueting N. Sex differences in reflex tachycardia induced by hypotensive drugs in unanesthetized rats. Am J Physiol 1975; 229: 652-656.

13 Silva CA, Portaro FC, Fernandes BL, lanzer DA, Guerreiro JR, Gomes CL, Konno K, Serrano SM, Nascimento N, Camargo AC. Tissue distribution in mice of BPP 10c, a potent prolinerich anti-hypertensive peptide of Bothrops jararaca. Toxicon 2008; 51: 515-523.

14 Lameu C, Hayashi MA, Guerreiro JR, Oliveira EF, Lebrun I, Pontieri V, Morais KL, Camargo $\mathrm{AC}$, Ulrich $\mathrm{H}$. The central nervous system as target for antihypertensive actions of a proline-rich peptide from Bothrops jararaca venom. Cytometry A 2010; 77: 220-230.

15 Hayashi J, Takeda K, Kuwabara T, Takesako T, Itoh H, Hirata M, Tanabe S, Nakata T, Sasaki S, Nakagawa M. Clonidine improves central attenuation og the baroreflex in spontaneously hypertensive rats. Jpn Heart J 1993; 34: 333-339.

16 Szabo B, Fritz T, Wedzony K. Effects of imidazoline antihypertensive drugs on sympathetic tone and noradrenaline release in the prefrontal cortex. $\mathrm{Br} \mathrm{J}$ Pharmacol 2001; 134: 295-304.

17 La Rovere MT, Pinna GD, Raczak G. Baroreflex sensitivity: measurement and clinical implications. Ann Noninvasive Electrocardiol 2008; 13: 191-207.
18 Pontieri V, Venezuela MK, Scavone C, Michelini LC. Role of endogenous nitric oxide in the nucleus tractus solitarii on baroreflex control of heart rate in spontaneously hypertensive rats. J Hypertens 1998; 16: 1993-1999.

19 Gomes CL, Konno K, Conceicao IM, lanzer D, Yamanouye N, Prezoto BC, Assakura MT, Rádis-Baptista G, Yamane T, Santos RA, de Camargo AC, Hayashi MA. Identification of novel bradykinin-potentiating peptides (BPPs) in the venom gland of a rattlesnake allowed the evaluation of the structure-function relationship of BPPs. Biochem Pharmacol 2007; 74: 1350-1360.

20 Raizada MK, Lu D, Sumners C. AT1 receptors and angiotensin actions in the brain and neuronal cultures of normotensive and hypertensive rats. Adv Exp Med Biol 1995; 377: 331-348.

21 Martins AH, Resende RR, Majumder P, Faria M, Casarini DE, Tárnok A, Colli W, Pesquero JB, Ulrich $\mathrm{H}$. Neuronal differentiation of P19 embryonal carcinoma cells modulates kinin B2 receptor gene expression and function. J Biol Chem 2005; 280: 19576-19586.

22 Feelisch M, Rassaf T, Mnaimneh S, Singh N, Bryan NS, Jourd'Heuil D, Kelm M. Concomitant $\mathrm{S}-, \mathrm{N}-$, and heme-nitros(yl)ation in biological tissues and fluids: implications for the fate of NO in vivo. FASEB J 2002; 16: 1775-1785.

23 Livak KJ, Schmittgen TD. Analysis of relative gene expression data using real-time quantitative PCR and the 2(-Delta Delta C(T)) method. Methods 2001; 25: 402-408.

24 Irigoyen MC, Lacchini S, De Angelis K, Michelini LC. Fisiopatologia da hipertensão: 0 que avançamos? Ver Soc Cardiol Estado de São Paulo 2003; 1: 20-45.

25 Thastrup O, Dawson AP, Scharff O, Foder B, Cullen PJ, Drobak BK, Bjerrum PJ, Christensen SB, Hanley MR. Thapsigargin, a novel molecular probe for studying intracellular calcium release and storage. Agents Actions 1989; 27: 17-23.

26 Krieger EM, Da Silva GJ, Negrão CE. Effects of exercise training on baroreflex control of the cardiovascular system. Ann N Y Acad Sci 2001; 940: 338-347.

27 Patel KP, Li YF, Hirooka Y. Role of nitric oxide in central sympathetic outflow. Exp Biol Med (Maywood) 2001; 226: 814-824.

28 Hauser GJ, Dayao EK, Zukowska-Grojec Z. Effect of pentobarbital anesthesia on the pressor response to agonists in vivo in normal and endotoxemic rats. Res Commun $\mathrm{Mol}$ Pathol Pharmacol 1995; 90: 289-300.

29 Lanfranchi PA, Somers VK. Arterial baroreflex function and cardiovascular variability: interactions and implications. Am J Physiol Regul Integr Comp Physiol 2002; 283: R815-R826.

30 Ducher M, Fauvel JP, Cerutti C. Risk profile in hypertension genesis: a five-year followup study. Am J Hypertens 2006; 19: 775-780.

31 Xing-Sheng Y, Yong-Zhi L, Jie-Xin L, Yu-Qing G, Zhang-Huang C, Chong-Fa Z, Zhi-Zhong $\mathrm{T}$, Shu-Zheng $\mathrm{L}$. genetic influence on baroreflex sensitivity in normotensive young men. Am J Hypertens 2010; 23: 655-659.

32 Bristow JD, Honour AJ, Pickering GW, Sleight P, Smyth HS. Diminished baroreflex sensitivity in high blood pressure. Circulation 1969; 39: 48-54.

33 Grassi G, Trevano FQ, Seravalle G, Scopelliti F, Mancia G. Baroreflex function in hypertension: consequences for antihypertensive therapy. Prog Cardiovasc Dis 2006; 48: 407-415.

34 Gordon FJ, Sved AF. Neurotransmitters in central cardiovascular regulation: glutamate and GABA. Clin Exp Pharmacol Physiol 2002; 29: 522-524.

35 Parmer RJ, Cervenka JH, Stone RA. Baroreflex sensitivity and heredity in essential hypertension. Circulation 1992; 85: 497-503.

36 Lameu C, de Camargo AC, Faria M. L-arginine signalling potential in the brain: the peripheral gets central. Recent Pat CNS Drug Discov 2009; 4: 137-142.

37 Nakamura H, Saheki T, Ichiki H, Nakata K, Nakagawa S. Immunocytochemical localization of argininosuccinate synthetase in the rat brain. J Comp Neurol 1991; 312: 652-679.

38 Kishi T, Hirooka Y, Sakai K, Shigematsu H, Shimokawa H, Takeshita A. Overexpression of eNOS in the RVLM causes hypotension and bradycardia via GABA release. Hypertension 2001; 38: 896-901.

39 Hirooka $Y$, Sakai K, Kishi T, Ito K, Shimokawa H, Takeshita A. Enhanced depressor response to endothelial nitric oxide synthase gene transfer into the nucleus tractus solitarii of spontaneously hypertensive rats. Hypertens Res 2003; 26: 325-331.

40 Kishi T, Hirooka Y, Kimura Y, Sakai K, Ito K, Shimokawa H, Takeshita A. Overexpression of eNOS in RVLM improves impaired baroreflex control of heart rate in SHRSP. Rostral ventrolateral medulla. Stroke-prone spontaneously hypertensive rats. Hypertension 2003; 41: 255-260. 